\title{
Smart materials for increasing the success of protein crystallization
}

Lata Govada ${ }^{1}$, Emmanuel Saridakis ${ }^{2}$, Sean Kassen ${ }^{1}$, Sahir Khurshid ${ }^{1}$, Hazim El-Sharif ${ }^{3}$, Subrayal Reddy ${ }^{3}$, Naomi Chayen ${ }^{1}$

${ }^{1}$ Imperial College London, London, United Kingdom, ${ }^{2}$ National Center for Scientific Research Demokritos, Agia Paraskevi 15310, Athens, Greece, ${ }^{3}$ University of Surrey, Guidford, United Kingdom

E-mail: I.govada@imperial.ac.uk

A new class of nucleants has emerged with the discovery that porous materials with a distribution of pore sizes similar to those of protein molecules are effective for inducing protein crystals. Building on the success of such porous materials, we sought to design nucleants with improved specificity for protein molecules while maintaining the idea of harnessing pores. Molecularly imprinted polymers (MIPs) also referred to as "smart materials" were created by imprinting a protein in a polymer and then removing it, leaving behind specific cavities which would then rebind this protein when introduced into a crystallization trial. These MIPs increased the number of crystal leads obtained from screening by $8-10 \%$ and significantly improved crystal quality)4. A new generation of MIPs suitable for high-throughput trials has recently been commercialised.

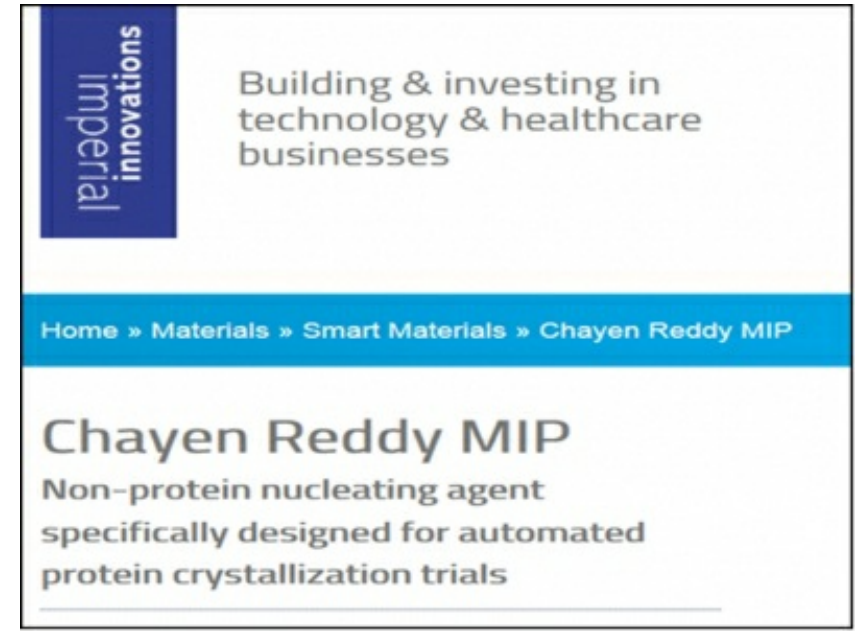

Keywords: Crystallization, proteins,MIPs 